\title{
Comparative Analysis and Evaluation of Common Spectrum Correction Methods
}

\author{
Meichen Liu ${ }^{1}$, Xu Liu ${ }^{1}$, Mei Li ${ }^{* 1}$ and Wei Wei ${ }^{2}$ \\ ${ }^{1}$ School of Information Engineering University of Geosciences (Beijing), Beijing, China \\ 2 School of Computer Science and Engineering University of Technology, Xi'an, China \\ maggieli@cugb.edu.cn
}

Keywords: frequency analysis; frequency spectrum correction; evaluation system.

\begin{abstract}
Although there are many spectrum correction theories and implementations, they are individual to specific problem and lack of a comprehensive comparison. This brings down their practical value in engineering applications. To solve this problem, we hereby establish a spectrum analysis and correction system. Each method i.e. Energy BaryCenter Method, Ratioing, Phase Difference Method, Continuous Zoom and All-Phase Spectrum Analysis. Meanwhile summarizing their accuracy,universality,practicability and window function. Then, we obtained a comprehensive assessment of all kinds of methods and created reference value for practical engineering application.
\end{abstract}

\section{Introduction}

During the digital signal processing, as the computer can only calculating for finite sample. Both Fourier transform and spectral analysis are focusing on analyzing the time domain truncation signal. Energy leak will happen when it comes to time domain truncation, and it makes the peak of frequency spectrum decreased, phases deviated, and precision reduced. Spectrum magnitude, frequency, and phase are not work at the application in the practical engineering even with the method of windowing. Thus we need to revise the amplitude, frequency, and phase of frequency spectrum. Common spectrum correcting methods can be divided into five kinds including all phase spectrum correction method found recent years: Firstly, Energy BaryCenter Method ${ }^{[1][2]}$. Secondly, Ratioing ${ }^{[3] .}$ Thirdly, Phase Difference Method [4]. Fourthly, Continuous Zoom ${ }^{[5][6] . ~ F i f t h l y, ~ a n d ~}$ All-Phase Spectrum Analysis ${ }^{[7][8] .}$

Some of the existing theory and application of spectrum correction methods are mature. They also have some research achievements in different ways of comprehensive analysis. As early as 2000, Kang Ding and Xiao-fei Zhan made a summary on the theoretical development of spectrum correction ${ }^{111]}$.In 2001, Xiao-yong Zhu and Kang Ding published Comprehensive Comparison of Discrete Spectrum Correction Method ${ }^{[6]}$. In 2006, Xiang-dong Huang made a summary based on all phase thought energy center of gravity method, the all phase ratio method and all phase method of phase difference correction method ${ }^{[18]}$. In 2008, Kang Ding, Ming Xie, and Zhi-jian Yang sorted out the updated theory and technology from the Discrete Spectrum Analysis and the Correction Theory and Technolory. In 2012, Yu-wen Mao, Ya-qing Tu, Wei Xiao, and Hui-yue Yang Aimed at the research progress in discrete density frequency room analyzing and correction method made a conclusion. Comprehensive comparison and analysis results we have thorough concluded from the proposing of existing spectrum correction method theory to mature, as well as the induction of technology. However, as the diversity, particularity, and pertinence of spectrum correction method, technician needs to analyze a certain number of parameters even through probation to ensure the exact spectrum correction method at the application in the practical engineering. The comprehensive comparison of pure theory and technology can't meet engineering application personnel's need. To solve it, we should found a set of assessment system which has practical reference meaning in engineering application that based on several common correction methods of parameters such as accuracy, applicability and feasibility. It has practical significance to engineering personnel when they make quickly judge and select at the appropriate spectrum 
correction method according to the comparative analysis and evaluation of different spectrum correction method.

\section{Comparison and Analysis of Common Spectrum Correction Method}

Energy BaryCenter Method. Energy BaryCenter Method is a universal correction method, which is through the use of various window functions, the characteristics that the infinite approximation of the discrete spectrum of origin energy center of gravity to make the discrete spectrum correction, and suitable for the frequency of the practical engineering applications of small fluctuations. The main parameters of the energy center of gravity method: the type of window function, the signal to noise ratio and the normalized frequency deviation, and the correction points.

Ratioing. Using the window spectrum ratio that near the main lobe peak signal of the two lines, which is namely that the ratio of the maximum amplitude of the main valve Ak and sharp value of $\mathrm{Ak}^{\prime}\left(\mathrm{k}^{\prime}\right.$ corresponds to the spectral line numberAk'), to establish a normalized frequency correction which is based on the variable equation, and then solving the, and making frequency, amplitude and phase correction. The amplitude accuracy obtained by the ratio correction method is still very high. The main parameters that affect the calibration accuracy of the ratio method: signal to noise ratio, frequency correction, window function type, etc.

Phase Difference Method. The method is through the time shift and the addition of different symmetrical windows for the twice FFT analysis, and the use of discrete spectrum corresponding to the phase difference of the peak spectral line to get the frequency and phase correction. On the FFT analysis of two time domain sequences with different window functions as $\mathrm{N}$ points and $\mathrm{M}$ points, the time domain frequency shift length is L, according to N, M, and L can be divided into two kinds of phase difference method in time domain translation and change window length method. The main parameters that affect the correction accuracy of the phase difference method: the time domain translation length $\mathrm{L}$, the frequency spectrum analysis, the interception length $\mathrm{N}$ and $\mathrm{M}$, the window length, and the window function type.

Continuous Zoom. In a wideband signal, the narrow band signal is analyzed in a frequency domain, and using FFT to estimate the spectrum of the signal, obtained the detailed range of narrow band spectrum; aiming at the locality that needed to refine to use the continuous improvement of the FFT to make operation, so as to get a local refinement of the high accuracy of the spectrum. This method can greatly improve the frequency resolution and improve the accuracy of the amplitude and phase of the sampling length without increasing the sampling length. The main parameters affected the accuracy of spectral continuous refining method of correction: the thinning ratio and the frequency resolution.

All- Phase Spectrum Analysis Method. The all phase idea refers to the FFT spectrum analysis in the time domain not only considers a kind of input signal segmentation; it can be included in the FFT spectrum analysis and has a very good inhibitory spectral leakage performance and "phase invariance", widely used in the spectrum analysis of the spectrum with high accuracy requirements of the spectrum analysis. The main parameters affected the accuracy of all phase spectrum correction: the sampling points, the window function type, and the size of the added noise.

Comparative Analysis. The energy center of gravity method, the ratioing, phase difference method is generally used to contain a single frequency component or the spacing between the larger multiple frequency components of signal of discrete spectrum correction method; continuous zoom of Fourier transforms, all phase spectrum analysis is generally used to contain multiple dense frequency component of the discrete spectrum correction method. On the intervals of larger multiple of the frequency components of the signal discrete spectrum correcting methods and more intensive frequency components of a signal correction method for discrete spectrum to compare the advantages and disadvantages of the two types and illustrated in table 1 , table 2 . 
Table.1 Discrete spectrum correction method to deal with the signal that Interval is larger and has multiple frequency components

\begin{tabular}{|l|l|l|}
\hline & Advantage & disadvantage \\
\hline $\begin{array}{l}\text { Energy } \\
\text { Method }\end{array}$ & $\begin{array}{l}\text { Directly correction } \\
\text { multi average power spectrum, } \\
\text { Simple Algorithm, } \\
\text { Compute fastly, } \\
\text { Small errors on the accuracy, } \\
\text { Correction method for a variety of } \\
\text { symmetrical window function }\end{array}$ & $\begin{array}{l}\text { Correction accuracy } \\
\text { related to the window } \\
\text { function. } \\
\text { For too dense frequency } \\
\text { spectrum, } \\
\text { analysis accuracy is not } \\
\text { high }\end{array}$ \\
\hline Ratioing & $\begin{array}{l}\text { Suitable for all symmetrical window } \\
\text { function, } \\
\text { High correction accuracy, } \\
\text { This method is suitable for general } \\
\text { engineering and signal processing }\end{array}$ & $\begin{array}{l}\text { We need to know the } \\
\text { spectrum mode functions } \\
\text { Also not suitable for } \\
\text { dense frequency } \\
\text { component spectrum } \\
\text { analysis }\end{array}$ \\
\hline Phase difference & $\begin{array}{l}\text { Good commonality. } \\
\text { Do not need to obtain the modulus } \\
\text { of the window function. } \\
\text { Can achieve precise correction of } \\
\text { signal's frequency and phase, high } \\
\text { correction accuracy }\end{array}$ & $\begin{array}{l}\text { If the frequency } \\
\text { components are very } \\
\text { close, it will affect the } \\
\text { accuracy of the } \\
\text { correction even cannot be } \\
\text { corrected }\end{array}$ \\
\hline
\end{tabular}

Table.2 Discrete spectrum correction method to deal with the signal that has

More intensive frequency components

\begin{tabular}{|l|l|l|}
\hline & \multicolumn{1}{|c|}{ Advantage } & \multicolumn{1}{c|}{ disadvantage } \\
\hline $\begin{array}{l}\text { Continuous } \\
\text { Zoom }\end{array}$ & $\begin{array}{l}\text { After refinement output spectrum } \\
\text { frequency resolution is increased, } \\
\text { peaks separate and clear, the trends same } \\
\text { as the spectrum that near the original } \\
\text { center of the spectrum }\end{array}$ & Computationally intensive \\
\hline $\begin{array}{l}\text { All- phase } \\
\text { analysis } \\
\text { method }\end{array}$ & $\begin{array}{l}\text { Full phase correction method is suitable } \\
\text { for the case of distant intervals and } \\
\text { dense spectrum, especially for dense } \\
\text { spectrum }\end{array}$ & Applications of immature \\
\hline
\end{tabular}

\section{Assessment Spectrum Correction Methods}

For spectrum correction analysis, there are three major indicators, accuracy, reliability and universality. Accuracy shows observations, calculated, closeness between estimated value and true value. There are factors that affect the accuracy of Noise size, SNR, Normalized frequency deviation, Refinement multiples, The number of sampling points, The size of negative frequency[17] Reliability shows that whether to implement a spectrum correction method relies on the condition easily and accurately obtain, general digital signal processing dependent on hardware requirements. To consider the complexity and computation of the signal processing algorithms, if some kind of spectral analysis's operation is too big. In practical engineering applications, if hardware conditions cannot support a large amount of computation, the practical application is restricted. Universality shows some spectral correction method can be widely applied in similar engineering applications, spectrum correction method widely used in various dynamic signal analyzer and computerized testing system, analysis of rotating machinery vibration signals. 
Analysis of torsion vibration signal like engine. High precision frequency and amplitude calibration system. But in a certain type of application various methods has its own advantages, practical application needs to take all factors, compare and analyze various spectral correction methods, all factors of the various spectral correction methods were compared, or according to the results of the correction accuracy of the method you choose after use directly. But affect the efficiency and practical value. Analyze the universality of various spectral correction method can provide reference for practical engineering application. Current correction method areas can be divided into vibration, voice, image and noise. In the windowing processing, there are some commonly used window function like Rectangular window, Hamming window, Hanning, Blackman and so on. Different window functions has a great impact on the quality of the spectral function in the signal processing, Therefore, select Window function is particularly important in the signal processing. So it can be summarized in Table 3. Details are as follows:

Table.3 Evaluation system

\begin{tabular}{|l|l|}
\hline $\begin{array}{c}\text { First-level } \\
\text { indicators }\end{array}$ & Secondary indicators \\
\hline \multirow{4}{*}{ Accuracy } & Noise resistance \\
\cline { 2 - 3 } & Deviation of normalized frequency \\
\cline { 2 - 3 } & Refinement multiples \\
\cline { 2 - 3 } & Affection of sampling points \\
\cline { 2 - 3 } & Contribution of negative frequency \\
\cline { 2 - 3 } & Affection of energy coefficient \\
\cline { 2 - 3 } & Affection of MSE e \\
\cline { 2 - 3 } & Affection of frequency resolution \\
\hline \multirow{5}{*}{ Reliability } & Affection of input SNR \\
\cline { 2 - 3 } & Algorithm complexity \\
\cline { 2 - 3 } & Computation \\
\hline \multirow{5}{*}{$\begin{array}{l}\text { Wniversality } \\
\text { Wunction }\end{array}$} & Applicability of mechanical vibration \\
\cline { 2 - 3 } & Applicability of speech signal processing \\
\cline { 2 - 3 } & Applicability of image processing \\
\cline { 2 - 3 } & Applicability of denoising system \\
\hline & Affection of window function type \\
\cline { 2 - 3 } & Affection of the window function $\mathrm{n}$ \\
\cline { 2 - 3 } & Affection of Window length \\
\cline { 2 - 3 } & Affection of time-domain translation + change in length \\
\hline
\end{tabular}

Using a simple four-level evaluation method is excellent, good, fair, and poor to evaluate the system. No need to refer all secondary indicators.

\section{Conclusion}

The System is based on theoretical basis of various spectral correction methods to undertake a comprehensive induction. Energy BaryCenter Method, Ratioing, Phase Difference Method, Continuous Zoom and All-Phase Spectrum Analysis that signal's frequency component has a larger interval. Spectrum analysis of continuous refinement and all phase spectrum correction method applies for the discrete spectrum correction that signals has multiple intensive frequency components. This article compares the advantages and disadvantages of various methods of correction, meanwhile summarized the critical parameter index that affect the accuracy of each spectrum correction method. We designed an evaluation system from four aspects. According to the assessment system design methodology, Accuracy, Reliability, Universality and Window 
function as a first-level metrics. On this basis, Split first-level indicators into specific secondary indicators. In practical applications, assess some kind spectrum correction method, then according to the secondary indicators and scoring screening, finally, determined the most appropriate spectral correction method.

\section{Acknowledgements}

This work is financially supported by the National Natural Science Foundation of China (Grant No. 41374185).

The authors appreciate the support of Jinqiao Seed Fund of Beijing Associationof Science and Technology.

\section{References}

[1] DING kang, Jiang Li-qi. Gravity correction method of discrete energy spectrum [J]. Journal of Vibration Engineering, 2001, 14(3): 354-358.

[2] DING Kang, ZHENG Chun-song, Yang Zhi-jian. Frequency Estimation Accuracy Analysis and Improvement of Energy Barycenter Correction Method for Discrete Spectrum [J]. Journal of Mechanical Engineering 2010, 46(5).

[3] XU Chuan-yan, DING Kang, YANG Zhi-jian. Influence on amplitude and phase estimation accuracy by interpolation method for discrete spectrum[J]Journal of Vibration Engineering2011, 24(6): 633-638.

[4] DING Kang,ZHONG Shun-cong. Universal discrete spectral phase difference correction method [J]. Journal of Electronics, 2003, 31(1): 142-145.

[5] MAO Yu-wen, TU Ya-qing, XIAO Wei. Research progress of detailed analysis and correction of discrete spectral density [J]. Vibration and Shock, 2012, 31(21): 112-119.

[6] DING Kang, ZHU Wing-ying, YANG Zhi-jian. Parameter Estimation Accuracy of FFT and FT Discrete Spectrum Correction[J] Journal of Mechanical Engineering,2010, 46(7): 68-73.

[7] HUANG Xiang-dong, WANG Zhi-hua. Principle of all-phase DFT restraining Spectral Leakage and application in Correcting Spectrum [J]. Jounral of Tianjin University, 2007, 40(7): 882-886.

[8] TAN Si-wei, REN Zhi-liang, SUN Chang-cun. Improvement of phase difference correcting spectrum method based on all-phase FFT[J]. systems Engineering and Electronics 2013, 35(1).

[9] Yangru X I E Y H M Z. The diagnosis method of bearing faults based on all phase FFT[J]. 2008.

[10]WU Guo-qiao , WANG Zhao-hua, HUANG Xiao-hong . All phase correction method for discrete spectrum [J]. Jounral of Data Acquisiting \&Processing, 2005, 20(3): 286-290.

[11]DING Kang,ZHANG Xiao-fei Development of spectrum correction theory [J]. Journal of Vibration Engineering, 2000, 13(1): 14-32.

[12] HAUNG Yun-zhi,XU Ke-jun. Spectrum correction method application based on FFT[J]. Journal of Scientific Instrument, 2001, 22(3): 80-81.

[13]CAO Yi,DING Kang,YANG Zhi-jian. The analysis of anti-noise performance of correcting methods on discrete spectrum [J]. Journal of Manufacturing Automation. 2011, 33(13): 40-43.

[14] XIE Ming. Phase correction method for phase and frequency correction in spectrum analysis [J]. Journal of Vibration Engineering, 1999, 12(4): 454-459.

[15]DING Kang,ZHU Xiao-yong. A Phase Difference Correcting Method on Discrete Spectrum Adapting to Any Window Function [J]. Journal of Electronics, 2001, 29(7): 987-989. 
[16]DING Kang,LUO Jiang-kai,XIE Xiao-ming. Time-Shifting Correcting Method of Phase Difference on Discrete Spectrum [J]. APPLIED MATHEMATICS AND MECHANICS2002, 23(7): 729-735.

[17] MAO Yu-wen,TU Ya-qing,ZHANG Hai-tao. Advances and Trendsin Spectrum Analysis Methodology with Negative Frequency Contribution[J]. Electrical Measurement \& Instrumentation, 2011, 48(5): 27-32.

[18] HUANG Xiang-dong. All Phase Digital Signal Processing [D]. Tianjin University PhD thesis, 2006

[19] YANG Zhi-jian. Analysis and correction of discrete spectrum theory and technology [M]. Science Press, 2008.

\section{Authors' background}

\begin{tabular}{|c|c|c|c|}
\hline Your Name & Title* & Research Field & Personal website \\
\hline LIU Mei-chen & Undergraduate & Signal Processing and Data Mining & $980661067 @ q q . c 0 m$ \\
\hline LIU Xu & Master & Signal Processing and Data Mining & maggieli@cugb.edu.cn \\
\hline LI Mei & $\begin{array}{c}\text { Ph.D., } \\
\text { Associate Professor, } \\
\text { Master's Instructor }\end{array}$ & $\begin{array}{c}\text { Engaged in teaching and research work } \\
\text { on Signal Processing }\end{array}$ & \\
\hline WEI Wei & Lecturer & $\begin{array}{c}\text { Engaged in teaching and research work } \\
\text { on Signal Processing }\end{array}$ & \\
\hline
\end{tabular}

